\title{
Imaging Cooper pairing of heavy fermions in $\mathrm{CeColn}_{5}$
}

\author{
M. P. Allan ${ }^{1,2,3,4 \dagger}$, F. Massee ${ }^{1,2 \dagger}$, D. K. Morr ${ }^{5 \star}$, J. Van Dyke ${ }^{5}$, A. W. Rost ${ }^{2,3}$, A. P. Mackenzie ${ }^{3,6}$, \\ C. Petrovic ${ }^{1}$ and J. C. Davis ${ }^{1,2,3,7 \star}$
}

The Cooper pairing mechanism of heavy fermion superconductors ${ }^{1-4}$, long thought to be due to spin fluctuations $^{5-7}$, has not yet been determined. It is the momentum space (k-space) structure of the superconducting energy gap $\Delta(k)$ that encodes specifics of this pairing mechanism. However, because the energy scales are so low, it has not been possible to directly measure $\Delta(k)$ for any heavy fermion superconductor. Bogoliubov quasiparticle interference imaging $^{8}$, a proven technique for measuring the energy gaps of superconductors with high critical temperatures ${ }^{9-11}$, has recently been proposed ${ }^{12}$ as a new method to measure $\Delta(k)$ in heavy fermion superconductors, specifically $\mathrm{CeColn}_{5}$ (ref. 13). By implementing this method, we detect a superconducting energy gap whose nodes are oriented along $k \|( \pm 1, \pm 1) \pi / a_{0}$ directions ${ }^{14-17}$. Moreover, for the first time in any heavy fermion superconductor, we determine the detailed structure of its multiband energy gaps $\Delta_{i}(k)$. For $\mathrm{CeColn}_{5}$, this information includes: the complex band structure and Fermi surface of the hybridized heavy bands, the fact that largest magnitude $\Delta(k)$ opens on a high-k band so that the primary gap nodes occur at unforeseen k-space locations, and that the Bogoliubov quasiparticle interference patterns are most consistent with $d_{x^{2}-y^{2}}$ gap symmetry. Such quantitative knowledge of both the heavy band-structure and superconducting gap-structure will be critical in identifying the microscopic pairing mechanism of heavy fermion superconductivity.

The heavy fermion superconductor $\mathrm{CeCoIn}_{5}$ (ref. 13) has a crystal unit cell with $a=b=4.6 \AA, c=7.51 \AA$, as shown schematically in Fig. 1a, and a superconducting critical temperature $T_{\mathrm{c}}=2.3 \mathrm{~K}$. If antiferromagnetically ordered, the $\mathrm{Ce}^{3+}$ atoms would exhibit local magnetic moments $\mu=0.15 \mu_{\mathrm{B}}$ (ref. 18). Although that state does not exist in the pure compound studied here, antiferromagnetic spin fluctuations do persist ${ }^{19}$. Pioneering research on $\mathrm{CeCoIn}_{5}$, using the recently introduced heavy fermion interference imaging technique ${ }^{20,21}$, reveals a heavy band ${ }^{22}$ at $T \approx 20 \mathrm{~K}$, in qualitative agreement with angle resolved photoemission measurements at similar temperatures ${ }^{23,24}$. However, no detailed and quantitative determinations of the heavy fermion band structures or Fermi surfaces have been reported for this compound. In its superconducting phase, the Cooper pairs are spin singlets ${ }^{25,26}$, so that an even parity $\Delta(\mathbf{k})$ is required. The magnetic field-angle dependence of thermal conductivity ${ }^{15}$ and specific heat ${ }^{17}$ are interpreted as evidence of energy-gap nodes $|\Delta(\mathbf{k})|=0$ for momentum space directions $\mathbf{k} \|( \pm 1, \pm 1) \pi / a_{0}$. A fully detailed knowledge of the $\mathbf{k}$-space structure of $\Delta(\mathbf{k})$ is required to understand the microscopic Cooper pairing mechanism of heavy fermions. This cannot be achieved using such indirect methods, or by using photoemission because the energy resolution required is $\delta E<100 \mu \mathrm{eV}$. Motivated thus, high-resolution Bogoliubov quasiparticle scattering interference imaging has recently been mooted $^{12}$ as a promising approach for determining $\Delta(\mathbf{k})$ of heavy fermion superconductors, specifically for $\mathrm{CeCoIn}_{5}$.

There are three elements of $\mathbf{k}$-space electronic structure expected in a generic heavy fermion superconductor ${ }^{4}$. First, the hightemperature state consists of a conventional (light) electronic band, indicated schematically by the dashed curve in Fig. 1b, that coexists with localized $f$-electron states on each magnetic atom. At lower temperatures, hybridization between this light band and the $f$-electron states results in its splitting into two new heavy bands, as shown schematically by the solid blue lines in Fig. 1b. The right panel shows how the resulting very flat bands generate a greatly enhanced density-of-electronic-states $N(E)$ within a few meV of $E_{\mathrm{F}}$-hence the 'heavy' effects seen in thermodynamic studies. At least one of these heavy bands crosses $E_{\mathrm{F}}$ at the new Fermi wave vector $\mathbf{k}_{\mathrm{F}}^{\mathrm{H}}$, as shown within the green box in Fig. 1b. It is in this region of k-space that, at even lower temperatures, the heavy quasiparticles are hypothesized to bind into heavy Cooper pairs. An energetically particle-hole symmetric superconducting energy gap $\Delta(\mathbf{k})$, probably of an unconventional nature ${ }^{1-7}$, is then expected to open in the heavy quasiparticle spectrum at the Fermi surface, as shown schematically in Fig. 1c. The right panel shows the further expected changes in $N(E)$ for a nodal $\Delta(\mathbf{k})$.

To search for this sequence of phenomena, we use pure

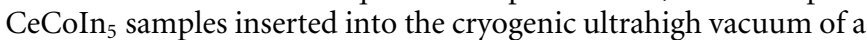
${ }^{3} \mathrm{He}$-refrigerator-based spectroscopic imaging scanning tunnelling microscope $^{27}$ (SI-STM), and mechanically cleaved therein. Atomically flat $a-b$ surfaces are achieved; a typical resulting topograph with visible atomic lattice $a_{0}=4.6 \AA$ (that was previously assigned to be the Ce lattice ${ }^{22}$ ) is shown in Fig. 1d. On all such surfaces, the density-of-states is determined from the spatially averaged differential tunnelling conductance $\langle\mathrm{d} I / \mathrm{d} V(E=\mathrm{eV})\rangle \propto \bar{N}(E)$ measured far from impurity atoms. Whereas the basic $\bar{N}(E)$ of the unhybridized 'light' bands is measured over the range $|E| \leq 200 \mathrm{meV}$ (Fig. 1e), the complex scattering interference features associated with the heavy band structure are only visible within the range $\sim-4 \mathrm{meV}<E<12 \mathrm{meV}$. Vertical arrows in Fig. 1f then indicate the limits of the hybridization gap $E_{\mathrm{h}}$ for $\mathrm{CeCoIn}_{5}$, as determined directly from the heavy band scattering interference analysis in Fig. 3.

\footnotetext{
${ }^{1}$ CMPMS Department, Brookhaven National Laboratory, Upton, New York 11973, USA, ${ }^{2}$ LASSP, Department of Physics, Cornell University, Ithaca, New York 14853, USA, ${ }^{3}$ School of Physics and Astronomy, University of St Andrews, St Andrews, Fife KY16 9SS, UK, ${ }^{4}$ Department of Physics, ETH Zurich, CH-8093 Zurich, Switzerland, ${ }^{5}$ Department of Physics, University of Illinois at Chicago, Chicago, Illinois 60607, USA, ${ }^{6}$ Max Planck Institute for Chemical Physics of Solids, D-01187 Dresden, Germany, ${ }^{7}$ Kavli Institute at Cornell for Nanoscale Science, Cornell University, Ithaca, New York 14853, USA. ${ }^{\dagger}$ These authors contributed equally to this work. *e-mail:dkmorr@uic.edu; jcseamusdavis@gmail.com
} 

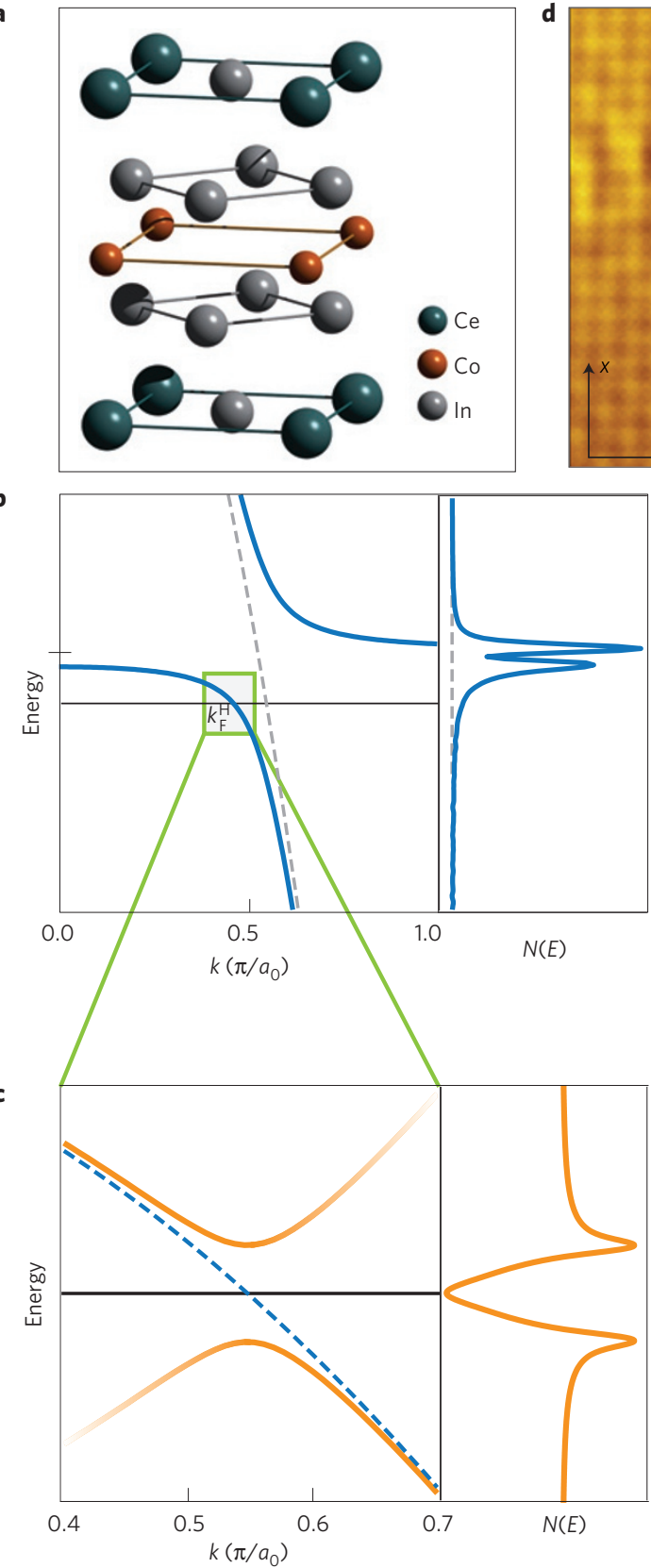

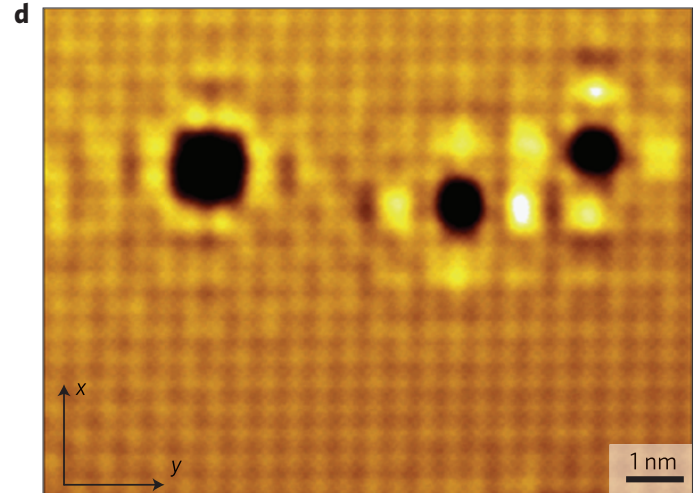

e

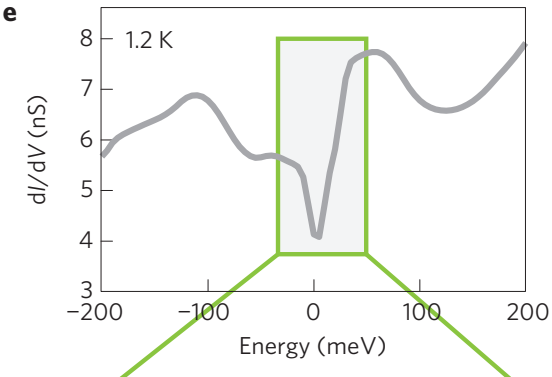

$\mathbf{f}$

g

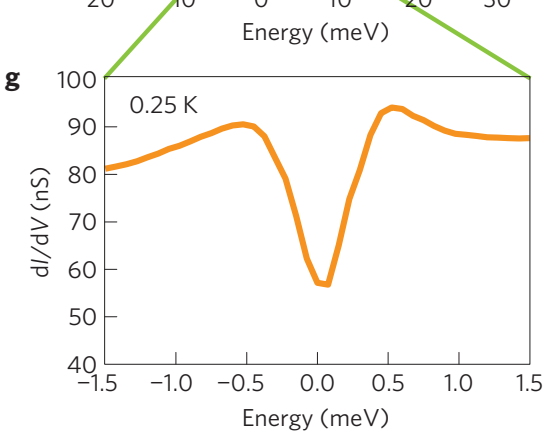

Figure 1 | Anticipated electronic structure of a heavy fermion superconductor. a, Schematic representation of the crystal unit cell of CeColn ${ }_{5}$. $\mathbf{b}$, Schematic of the typical evolution of the $\mathbf{k}$-space electronic structure observed ${ }^{21}$ as hybridization splits the light band into two heavy bands, and the consequential effects on the density of states $N(E)$. c, Schematic of expected evolution of the $\mathbf{k}$-space electronic structure as the superconducting energy gap appears (presumably) on one of the new heavy bands. The right-hand panel shows expected changes in the $N(E)$ due to heavy fermion Cooper pairing, here simulated for a $d$-wave symmetry energy gap. d, Topographic image of the termination surface of cryo-cleaved CeColn $n_{5}$ used in this study. $\mathbf{e}$, Average differential conductance spectra $g(E)$ in the energy range of light band(s) $|E| \leq 200 \mathrm{meV}$, measured using the lock-in technique with a bias modulation of $5 \mathrm{meV}$ so that any finer energetic features are unresolvable. Data in $\mathbf{f}, \mathbf{g}$ below are acquired with decreasing bias modulation compared to that in $\mathbf{e}$. f, Measured average differential conductance spectra in the energy range spanning the hybridization gap $\sim-4$ meV $<E<12$ meV. The hybridization gap $E_{\mathrm{h}}$ between vertical arrows is determined directly from heavy quasiparticle scattering interference (Fig. 3), measured with bias modulation of 1.5 meV so that any finer energetic features, for example the superconducting energy gap, are unresolvable. $\mathbf{g}$, Measured differential conductance spectra in the energy range spanning the superconducting gap $|E| \leq 600 \mu \mathrm{eV}$, measured with a bias modulation of $70 \mu \mathrm{eV}$ and a thermal energy resolution of $75 \mu \mathrm{eV}$. The colours associated with the three different energy scales in $\mathbf{e}-\mathbf{g}$ are used throughout the paper to indicate the equivalent energy scales.

On entering the superconducting phase, $\bar{N}(E)$ develops an energy gap with a maximum value $\left|\Delta_{\max }\right|=600 \pm 50 \mu \mathrm{eV}$, a Vshaped $\bar{N}(E) \propto E$ that is the signature of a nodal ${ }^{28,29} \Delta(\mathbf{k})$, and a finite $^{28-30} N(E=0)$, all as shown in Fig. 1g. Figure 2a shows a typical example of atomically resolved images $g(\mathbf{r}, E) \equiv \mathrm{d} I / \mathrm{d} V(r, E=\mathrm{eV})$ measured within the superconducting gap at $E=250 \mu \mathrm{eV}$, and acquired in a $32 \mathrm{~nm} \times 32 \mathrm{~nm}$ field of view (FOV). The superconducting peak-to-peak gap map $\Delta_{\mathrm{pp}}(r)$ in the same FOV (Fig. 2b) reveals the electronic homogeneity of this material. In Fig. 2c we show an image of the $\mathrm{CeCoIn}_{5}$ Abrikosov vortex array acquired at $T=250 \mathrm{mK}, B=3 \mathrm{~T}$ in a larger FOV; its shape and orientation are in excellent agreement with small-angle 

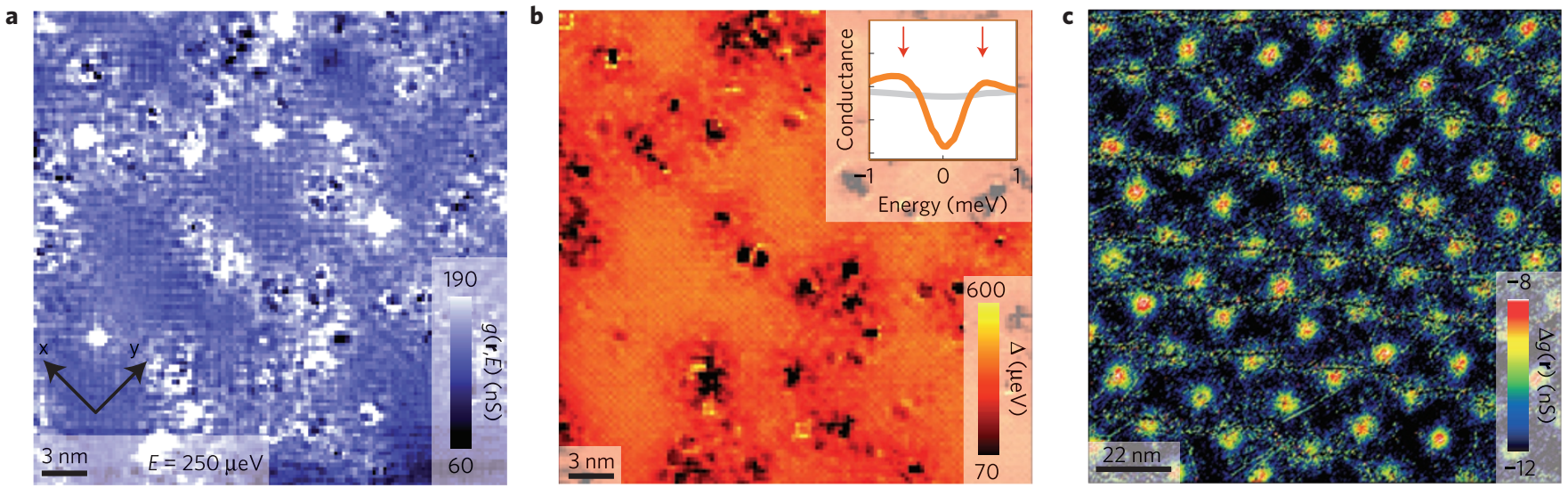

Figure 2 | Imaging superconducting gap map and vortex lattice of CeColn $\mathbf{5}$. a Typical example of $g(\mathbf{r}, E)$ measured below the superconducting gap edge $\left|\Delta_{\mathrm{pp}}\right|=550 \mu \mathrm{eV}$ and acquired in a $32 \mathrm{~nm} \times 32 \mathrm{~nm}$ FOV. b. Superconducting peak-to-peak gap map $\Delta_{\mathrm{pp}}(\mathbf{r})$ measured between the particle-hole symmetric peaks in $g(\mathbf{r}, E)$ taken in same FOV as $\mathbf{a}$. The homogeneity of the gap structure away from impurities is as expected in these pure materials. The inset shows a typical spectrum with arrows denoting the maximal gap, $\Delta_{\mathrm{pp}}$. c, Image of $\mathrm{CeColn}_{5} \Phi=h / 2 \mathrm{e}$ Abrikosov vortex array at $B=3 \mathrm{~T}$ by measuring $g(\mathbf{r}, E=0,3 \mathrm{~T})-g\left(\mathbf{r}, E=\Delta_{\mathrm{pp}}, 3 \mathrm{~T}\right)$. The lattice is consistent with the square lattice reported by neutron scattering experiments ${ }^{31}$, taking into account the small field drift.

neutron scattering studies ${ }^{31}$. As all these phenomena disappear at the superconducting $T_{\mathrm{c}}$ observed in bulk measurements, the $\left|\Delta_{\text {pp }}\right|=550 \pm 50 \mu \mathrm{eV}$ energy gap with $\mathrm{V}$-shaped $N(E)$ is definitely that of the superconductor. Determination of the $\mathbf{k}$-space structure of $\Delta(\mathbf{k})$ for $\mathrm{CeCoIn}_{5}$ is then the main focus of this paper.

To proceed, we image the differential conductance $g(\mathbf{r}, E)$ with atomic resolution and register, and then determine $g(\mathbf{q}, E)$, the square root of the power spectral density Fourier transform of each image. The measurements are all carried out at $250 \mathrm{mK}-$ thereby achieving an energy resolution $\delta E \sim 3.5 \mathrm{k}_{\mathrm{B}} \mathrm{T} \sim 75 \mu \mathrm{eV}$. By using a $>30 \mathrm{~nm}$-square FOV we simultaneously achieve $\mathbf{q}$-space resolution $|\delta q|<2 \%$ of the reciprocal unit cell, and a concomitant $\mathbf{k}$-space resolution $|\delta k|<2 \%$ of the Brillouin zone. To investigate both how the light bands transform to hybridized heavy fermion states, and how superconductivity then emerges, we measure these datasets on three distinct energy scales, each of about an order of magnitude smaller energy range than the previous one, as described in the Supplementary Information. These data are used to evaluate elements of $\mathbf{k}$-space electronic structure, based on the fact that elastic scattering of electrons with momentum $-\mathbf{k}(E)$ to $+\mathbf{k}(E)$ generates interference patterns occurring as maxima at $\mathbf{q}(E)=2 \mathbf{k}(E)$ in $g(\mathbf{q}, E)$, an effect recently revealed to exist even when hybridization generates heavy fermion bands $s^{20-22}$. In Supplementary Section SI and Fig. S1 we show the measured $g(\mathbf{q}, E)$ at $T=1.2 \mathrm{~K}$, for $-100 \mathrm{meV}<E<30 \mathrm{meV}$, focusing on the light unhybridized electronic structure. Here the maximum intensity features move slowly and smoothly to smaller $|\mathbf{q}|$-radii with increasing $E$, thereby revealing a light and simple tetragonal band (Supplementary Section SI and Movie S1).

Significant departures from this simple phenomenology are found to occur only within the energy range $\sim-4 \mathrm{meV}<E<12 \mathrm{meV}$. In Fig. 3a-e we next show the measured $g(\mathbf{q}, E)$ at $T=250 \mathrm{mK}$ within this range (Supplementary Section SI). The onset of hybridization is detected as a sudden transformation of the previously unchanging structure of $g(\mathbf{q}, E)$ occurring at $E \approx-4 \mathrm{meV}$ (Fig. 3b), followed by a rapid evolution of the maximum intensity features (indicated by circles and arrows Fig. 3c) towards smaller $|\mathbf{q}|$-radius interference patterns. Then, in Fig. 3c we see that an abrupt jump to a larger $|\mathbf{q}|$-radius occurs, followed by a second rapid diminution of interference pattern $|\mathbf{q}|$-radii in Fig. 3c-e (complete phenomena shown in Supplementary Section S1 and Movie S1). These are all the expected quasiparticle interference (QPI) signatures of the appearance of hybridized heavy fermion bands. Thus, for $\mathrm{CeCoIn}_{5}$ this approach reveals how the light conduction band is split into two heavy bands within the hybridization gap $-4 \mathrm{meV}<E_{\mathrm{h}}<12 \mathrm{meV}$. To see this directly, we show in Fig. 3f,g the measured evolution of the maxima in $g(\mathbf{q}, E)$ for two directions in $\mathbf{q}$-space. The light band (grey dots) begins to deviate near $-4 \mathrm{meV}$ towards the lower heavy band which crosses $E_{\mathrm{F}}$ at smaller $|\mathbf{q}|=2\left|\mathbf{k}_{\mathrm{F}}^{\mathrm{H}}\right|$, and evolves quickly to even smaller $|\mathbf{q}|$ (blue dots). Within a few $\mathrm{meV}$ above $E_{\mathrm{F}}$, the interference patterns jump to a much larger $|\mathbf{q}|$ and then evolve (blue dots) back towards the light band (grey dots), which they rejoin near $+12 \mathrm{meV}$. This heavy band actually crosses below $E=0$ at high $k$, producing an electron-like Fermi surface whose intra-band scattering interference generates interference patterns at low $\mathbf{q}$ (blue dots $E<0$ as $|\mathbf{q}| \rightarrow 0$ ). These data (Fig. 3a-e, Supplementary Section SI), and the extracted dispersions (Fig. 3f,g) are next used to determine details of the heavy fermion band structure.

In general for a complex and multi-band $\mathbf{k}$-space structure, achieving a deterministic inversion procedure from $g(\mathbf{q}, E)$ data to the complete band structure can be challenging ${ }^{11}$. Here, comparison of the predicted scattering interference dispersions $|\mathbf{q}(E)|$ from a specific model of the heavy bands described in Supplementary Section SII and Fig. 3h, with the experimental $|\mathbf{q}(E)|$ data within the hybridization range $E_{\mathrm{h}}$, reveals good agreement (Supplementary Figs S2 and S3). The critical elements in our model that lead to this agreement are the nearly parallel sections of the light band contours-of-constant-energy and the hybridization with a specifically shaped $f$-band. Some of these same elements of $\mathbf{k}$-space structure can equally be found in ref. 12. Our model concentrates on best emulation of the key empirical phenomena of the heavy QPI data. On this basis, the $g(\mathbf{q}, E)$ in Fig. 3a-g are used to motivate the detailed k-space model for the heavy bands of $\mathrm{CeCoIn}_{5}$, as shown in Fig. 3h (Supplementary Section SII). Here, within the range $E_{\mathrm{h}}$, a light-hole-like band centred around $\Gamma$ (or equivalently M) hybridizes with a localized $f$-electron band (Supplementary Section SII). The resulting lower heavy band $\beta$ has a simple Fermi surface and closes quickly above $E_{\mathrm{F}}$, whereas the upper heavy band $\alpha$ is highly anisotropic with a complex Fermi surface as it crosses below $E_{\mathrm{F}}$, making it effectively electron like. Our model indicates the possibility of a small dimple that crosses back above $E_{\mathrm{F}}$, but this is in no way critical to the subsequent analysis. The Fermi surfaces are shown as solid lines on the $E=0$ planes of Fig. 3h. Their relationship with the FS areas deduced from quantum oscillations (QO) at high fields ${ }^{32,33}$ is discussed in Supplementary Section VI. 


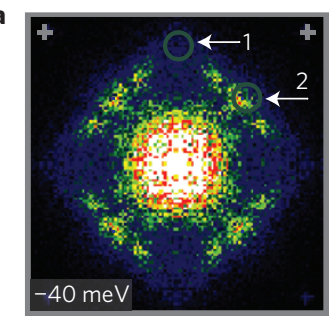

b
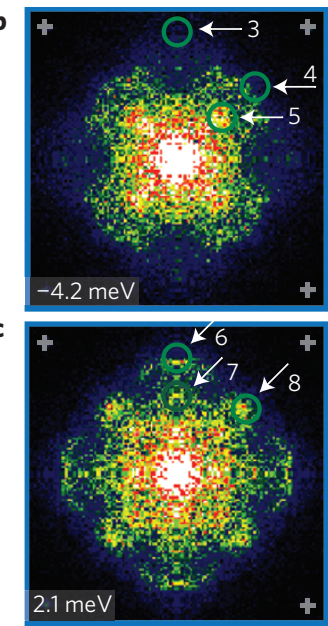

d

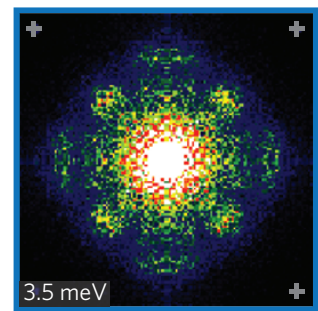

e

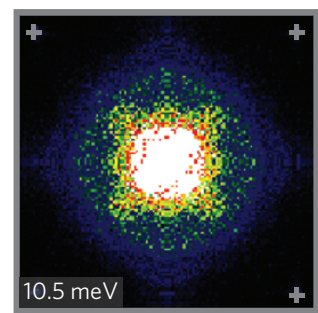

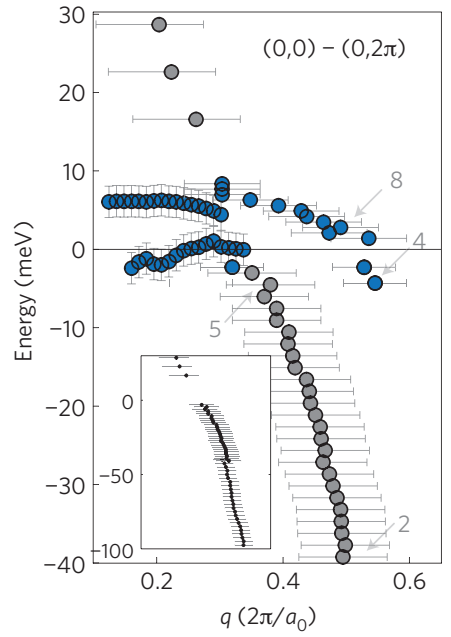

g

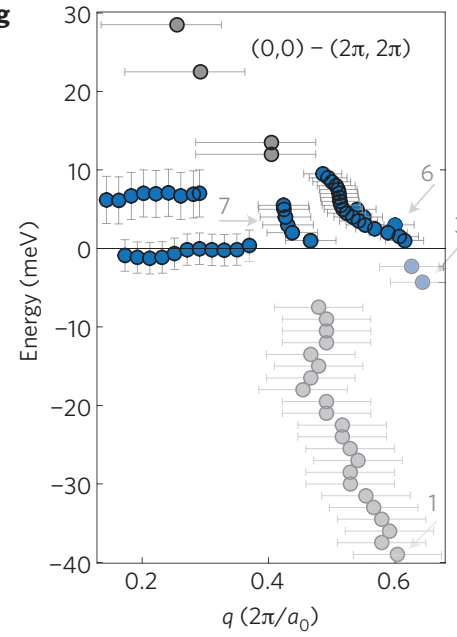

h
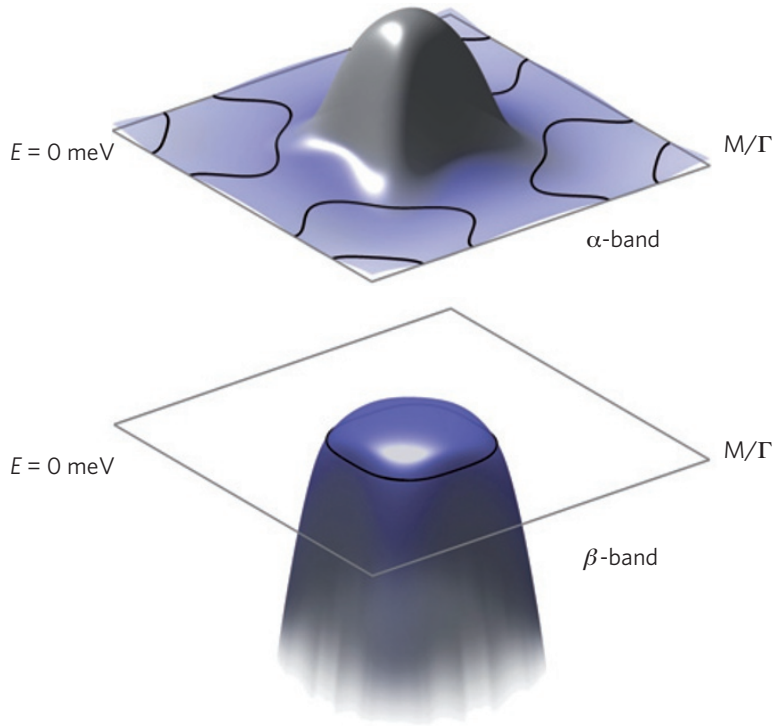

High Low

Figure 3 | Determination of heavy fermion bands and Fermi surfaces at $B=0$ in CeColn $\mathbf{n}_{\mathbf{5}}$. a-e, Measured $g(\mathbf{q}, E)$ at $T=250 \mathrm{mK}$ and $\delta E \sim 75 \mu \mathrm{eV}$, concentrating on the heavy-fermion-forming hybridization window $-4 \mathrm{meV}<E_{\mathrm{h}}<12 \mathrm{meV}$. The numbered arrows indicate locations of maxima in $g(\mathbf{q}, E)$ whose dispersion is identified using similarly numbered arrows in $\mathbf{f}, \mathbf{g}$ and grey crosses mark the Bragg peak locations. All $g(\mathbf{q}, E)$ data, except those measured within the superconducting gap, are treated as described in Supplementary Section SV. $\mathbf{f}, \mathbf{g}$, Measured evolution of the light band scattering interference dispersion $|\mathbf{q}(E)|$ (grey circles) and its transition to two heavy bands (blue circles) each with a distinct $|\mathbf{q}(E)|$. Some points are fitted on $g(\mathbf{q}, E=$ const) layers, whereas others are fitted from $g(|\mathbf{q}|, E)$ cuts (Supplementary Section SIV). The error bars are estimates based on the peak widths and the confidence intervals from the peak fits. $\mathbf{h}$, Momentum-space model for the hybridization-induced heavy bands and Fermi surfaces of CeColn ${ }_{5}$. Detailed parameterization is given in Supplementary Section SII. At the centre of the upper half of this panel we see the light band (grey) closing at the centre. As $E=0$ is approached from above, the upper heavy band (blue) diverges from the light band and begins to disperse very rapidly outwards, crossing $E=0$ at high $k$. The lower half of this panel shows the light band (grey) approach $E=0$ from below, beginning to diverge rapidly towards low $k$ as it crosses $E=0$ (blue), and then closing just above $E=0$. The characteristic Fermi surface areas deduced from the data/model for the heavy bands shown in Fig. 3 are somewhat comparable to those found in quantum oscillation studies in CeColn ${ }_{5}$ (refs 32,33). However, quantitative comparison of these to the FS areas determined by quantum oscillations at high field seems, in principle, to be quite challenging, because a rapid field-induced reorganization of the band-structure occurs above $B=4.5 \mathrm{~T}$. Furthermore, data on the CeColn 5 k-space structure measured using both SI-STM (ref. 22) and ARPES (refs 23,24) at $T \sim 20 \mathrm{~K}$ (energy resolution $\delta E \approx 3.5 \mathrm{k}_{\mathrm{B}} T \approx 5 \mathrm{meV}$ ) are not inconsistent with the higher precision heavy-band determinations herein; comparison with the quantum oscillations is discussed in Supplementary Section SVI.

To investigate the superconductivity on the heavy bands in Fig. 3h, Fig. 4a-e shows the measured $g(\mathbf{q}, E)$ at $T=250 \mathrm{mK}$ and $|E|<300 \mu \mathrm{eV}$, within the superconducting energy gap. Here we see extremely rapid evolution in $g(\mathbf{q}, E)$ over energies of a few $100 \mu \mathrm{eV}$, and the appearance of a four-fold symmetric 'nodal' $g(\mathbf{q}, E)$ structure as $E \rightarrow 0$. Clearly, this $g(\mathbf{q}, E=0)$ exhibits far more 
a

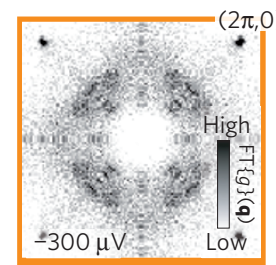

f

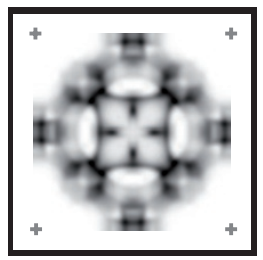

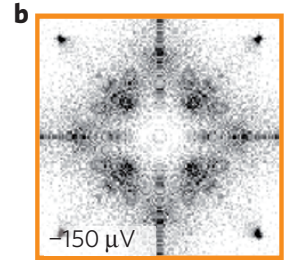
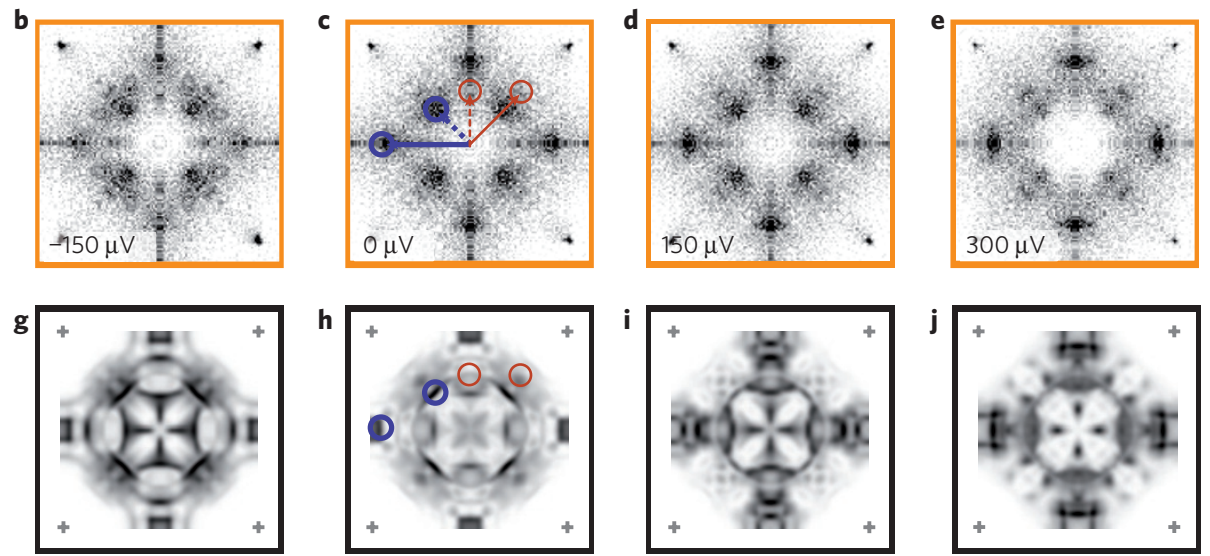

k

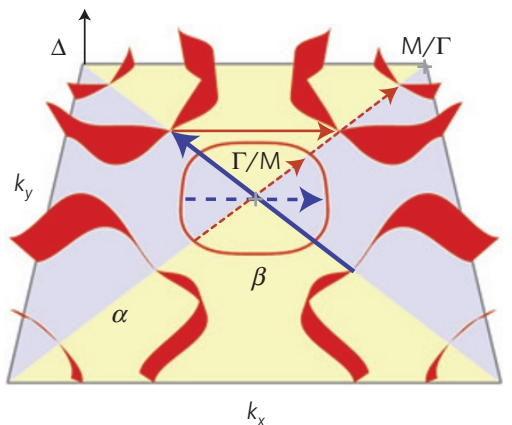

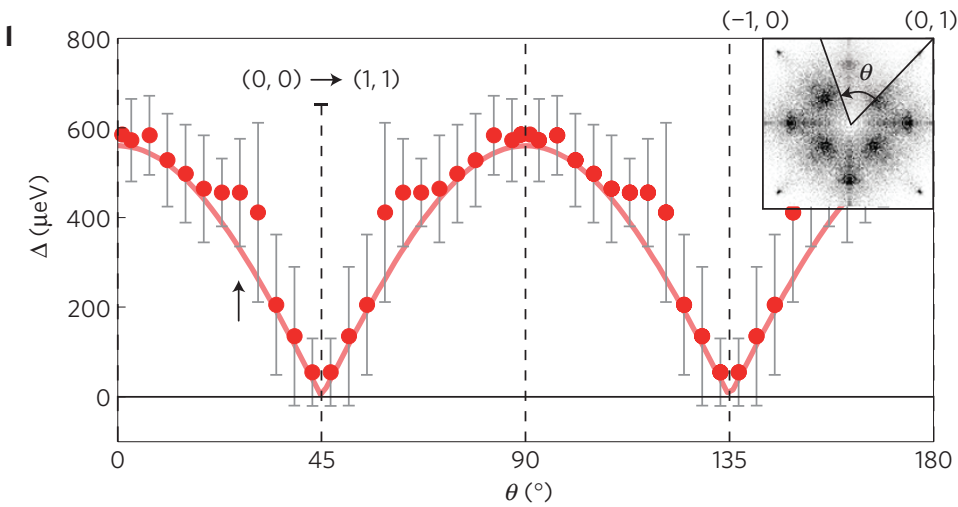

Figure 4 | Momentum-space superconducting energy gap $\boldsymbol{\Delta}(\mathbf{k})$ of CeColn $\mathbf{n}_{5}$. a-e, Measured $g(\mathbf{q}, E)$ at $T=250 \mathrm{mK}$ and $\delta E \sim 75 \mu \mathrm{eV}$, within the heavy fermion superconductivity energy window $-600 \mu \mathrm{eV}<E<600 \mu \mathrm{eV}$. The raw data were symmetrized to increase the signal-to-noise ratio. The Bragg peak location $(2 \pi, 0)$ is indicated in $\mathbf{a}$, and crosses mark the Bragg peak locations in $\mathbf{f}-\mathbf{j}$. $\mathbf{f}-\mathbf{j}$, Corresponding Bogoliubov QPI simulations of $g(\mathbf{q}, E)$ at the same energies as in a-e (Supplementary Section SII) on the two bands as shown in Figs $3 \mathrm{~h}, 4 \mathrm{k}$, and for superconducting gaps given approximately by $\Delta_{\beta}\left(\theta_{\mathrm{k}}\right)=0$ and a $d_{x^{2}-y^{2}}$ symmetry gap having $\Delta_{\alpha}\left(\theta_{k}\right)=A \cos \left(2 \theta_{k}\right)$ with $A=600 \mu \mathrm{eV}$. k, Fermi surfaces and energy gaps of CeColn 5 modelled using heavy QPI in Fig. 3 (Supplementary Section SII). The superconducting energy gaps $\Delta_{i}(k)$ used to achieve the most successful Bogoliubov QPI simulations are shown in red. Although a zero gap is shown on the $\beta$ sheet, our data only constrain $\Delta_{\beta}$ to be less than our energy resolution of $75 \mu \mathrm{eV}$; previous thermodynamic studies $^{13}$ show that both $\alpha$ and $\beta$ bands are gapped at the lowest temperatures. As in $\mathbf{c}$, the internodal scattering vectors consistent with the data are show as solid blue arrows while the Friedel oscillation wave vectors of the ungapped (at $250 \mathrm{mK}$ ) Fermi surface regions are shown as dashed blue lines (details in Supplementary Section SII). I, Measured $\left|\Delta\left(\theta_{q}\right)\right|$ using techniques as described in text (Supplementary Section SIII) and its comparison with the simplest multi-band gap structure $\Delta_{\beta}\left(\theta_{k}\right) \approx 0$ and $\Delta_{\alpha}\left(\theta_{k}\right)=A \cos \left(2 \theta_{k}\right)$ with $A=600 \mu \mathrm{eV}$ that we find to be consistent with all the Bogoliubov $g(\mathbf{q}, E)$ data herein. The arrow identifies the strong departures from this simple gap function. Error bars are estimates based on the peak widths and the confidence intervals from the peak fits, as described in Supplementary Section SV.

complexity than expected for a single-band nodal superconducting energy gap. To explore these phenomena we carry out Bogoliubov QPI simulations based on the two heavy bands, $\alpha$ and $\beta$ (Figs $3 \mathrm{~h}, 4 \mathrm{k}$ ), but now specifying their superconducting energy gaps $\Delta_{\alpha}(\mathbf{k})$ and $\Delta_{\beta}(\mathbf{k})$, whose derivation is discussed below. Here the inter-nodal scattering wave vectors for the $\alpha$ band (coloured arrows Fig. 4k) are demonstrably consistent with the measured inter-nodal scattering vectors in $g(\mathbf{q}, E=0)$ data, whereas the equivalent internodal signatures are undetected for the $\beta$ band. As specific heat data show that all the main bands in $\mathrm{CeCoIn}_{5}$ are gapped at lowest temperatures ${ }^{13}$, this suggests that the gap on the $\beta$ band, although extant, is too small to be detected at $T \sim 250 \mathrm{mK}$ (ref. 34) because the energy uncertainty of tunnelling electrons $\delta E \sim 75 \mu \mathrm{eV}$ probably exceeds the gap maximum. This situation also provides a simple and plausible explanation for the non-zero tunnelling conductance at $E=0$. What our data do indicate is that the primary gap of $\mathrm{CeCoIn}_{5}$ actually occurs on the high-k $\alpha$-band with lines of gap-nodes along the $\mathbf{k}=(0,0) \rightarrow( \pm \pi, \pm \pi) / a_{0}$ directions, so that the dominant gap nodes in CeCoIn ${ }_{5}$ occur at unforeseen $\mathbf{k}$-space locations (Fig. $4 \mathrm{k}$ ).

Next we consider a detailed comparison of the measured $g(\mathbf{q}, E)$ data for $|E| \leq 600 \mu \mathrm{eV}$ at $T \sim 250 \mathrm{mK}$ with theoretical simulations of
Bogoliubov QPI in $g(\mathbf{q}, E)$ using the $\alpha, \beta$ Fermi surfaces described in Figs $3 \mathrm{~h}, 4 \mathrm{k}$. The simulations have been carried out using various symmetries for the superconducting energy gaps and are described in full detail in Supplementary Section SII. Our model with superconducting gaps of $d_{x^{2}-y^{2}}$ symmetry given approximately by $\left|\Delta_{\beta}\left(\theta_{k}\right)\right|<50 \mu \mathrm{eV}$ and $\Delta_{\alpha}\left(\theta_{k}\right)=A \cos \left(2 \theta_{k}\right)$, with $A=600 \pm 50 \mu \mathrm{eV}$, yields a set of simulated $g(\mathbf{q}, E)$ that are far more consistent with the experimental data than any of the other models we have considered (Supplementary Fig. S5, Supplementary Section SII). For comparison, a direct experimental estimation of $\left|\Delta\left(\theta_{k}\right)\right|$ can be achieved by using $g(\mathbf{q}, E)$ data in a procedure largely independent of the Fermi surface details. To obtain the angle $\theta_{q}$ dependence of the energy gap that opens at $T_{\mathrm{c}}$, we integrate the total spectral weight $g(\mathbf{q}, E)$ within a given $|\delta q|$ range containing the Fermi surface, with the lowest $|\mathbf{q}|$ large enough to exclude effects of heterogeneity and the largest $|\mathbf{q}|$ small enough to exclude the Bragg peaks. A clear gap $\Delta\left(\theta_{q}\right)$ is observed to open in this integral of $g(\mathbf{q}, E)$ on passing below $T_{\mathrm{c}}$, as demonstrated in Supplementary Section SIII. In Fig. 41 we plot the measured energy gap $\left|\Delta\left(\theta_{q}\right)\right|$ from this technique (red dots) along with $\Delta_{\alpha}\left(\theta_{k}\right)=\left|A \cos \left(2 \theta_{k}\right)\right|$ with $A=600 \mu \mathrm{eV}$ (solid line). Their agreement provides strong independent motivation for our 
gap structure model (Fig. 4k and Supplementary Section SII). A final stimulating observation is that the departures in the $\Delta\left(\theta_{q}\right)$ at a higher energy from the simple $\Delta_{\alpha}\left(\theta_{k}\right)=A \cos \left(2 \theta_{k}\right)$ might be expected if high $\mathbf{q}$ scattering between these locations on the $\alpha$ band is involved in the Cooper pairing mechanism.

Overall, these data represent the first measurements of the $\mathbf{k}$-space structure of the superconducting energy gaps $\Delta_{i}(\mathbf{k})$ for any heavy fermion superconductor. They reveal a wealth of previously unknown information on the $\Delta_{i}(\mathbf{k})$ of $\mathrm{CeCoIn}_{5}$, including: the complex Fermi surface of the hybridized heavy bands (Figs $3 \mathrm{~h}, 4 \mathrm{k}$ ); the spectroscopic signature of four nodal lines in $|\Delta(\mathbf{k})|$ oriented along $\mathbf{k}=( \pm 1, \pm 1) \pi / a_{0}$ or Ce-Ce directions ${ }^{14-17}$; that the dominant $\Delta(\mathbf{k})$ opens on the $\alpha$ heavy band at high $\mathbf{k}$ (Fig. $4 \mathbf{k}$ ); the unforeseen $\mathbf{k}$-space locations of the dominant gap nodes that ensues (Fig. 4k); that the Bogoliubov QPI patterns are most consistent with $d_{x^{2}-y^{2}}$ gap symmetry; and evidence for a departure in $\Delta(\mathbf{k})$ from a simple $\cos \left(2 \theta_{k}\right)$ dependence at regions of the $\alpha$ band connected by $\mathbf{q} \approx( \pm 1, \pm 1) \pi / a_{0}$ (Fig. $\left.4 \mathrm{k}, 1\right)$. These detailed multiband $\Delta_{i}(\mathbf{k})$ data provide information critical for determining the microscopic mechanism of unconventional superconductivity in $\mathrm{CeCoIn}_{5}$. Moreover, the novel approach proposed in ref. 12, and established here, reveals an exciting new opportunity to achieve a quantitative understanding of the microscopic physics of heavy fermion superconductivity in general.

\section{Methods}

High-quality $\mathrm{CeCoIn}_{5}$ single crystals were grown at BNL (details in ref. 13). Magnetization measurements before sample insertion into the STM show a sharp transition with $T_{\mathrm{c}}=2.1 \mathrm{~K}$. The samples were mechanically cleaved in cryogenic ultrahigh vacuum at $T \sim 10 \mathrm{~K}$ and directly inserted into the STM head at $4.2 \mathrm{~K}$. Etched atomically sharp and stable tungsten tips with an energy-independent density of states are used. Differential conductance measurements throughout used a standard lock-in amplifier. See Supplementary Information for additional details on data treatment and extraction.

Received 18 March 2013; accepted 24 May 2013; published online 14 July 2013

\section{References}

1. Monthoux, P., Pines, D. \& Lonzarich, G. G. Superconductivity without phonons. Nature 450, 1177-1183 (2007).

2. Norman, M. R. The challenge of unconventional superconductivity. Science 332, 196-200 (2011).

3. Scalapino, D. J. A common thread: The pairing interaction for unconventional superconductors. Rev. Mod. Phys. 84, 1383-1417 (2012).

4. Coleman, P. in Handbook of Magnetism and Advanced Magnetic Material, Volume Fundamental Theory (eds Kronmüller, H \& Parkin, S) 95-148 (Wiley, 2007).

5. Miyake, K., Schmitt-Rink, S. \& Varma, C. M. Spin-fluctuation-mediated even-parity pairing in heavy-fermion superconductors. Phys. Rev. B 34, 6554-6556 (1986).

6. Scalapino, D. J., Loh, E. Jr \& Hirsch, J. E. $d$-wave pairing near a spin-density-wave instability. Phys. Rev. B 34, 8190-8192 (1986).

7. Beal-Monod, M. T., Bourbonnais, C. \& Emery, V. J. Possible superconductivity in nearly antiferromagnetic itinerant fermion systems. Phys. Rev. B 34, 7716-7720 (1986).

8. Wang, Q. H. \& Lee, D. H. Quasiparticle scattering interference in high-temperature superconductors. Phys. Rev. B 67, 020511 (2003).

9. Hoffman, J. E. et al. Imaging quasiparticle interference in $\mathrm{Bi}_{2} \mathrm{Sr}_{2} \mathrm{CaCu}_{2} \mathrm{O}_{8+\mathrm{d}}$. Science 297, 1148-1151 (2002).

10. Hanaguri, T. et al. Quasi-particle interference and superconducting gap in $\mathrm{Ca}_{2-x} \mathrm{Na}_{\mathrm{x}} \mathrm{CuO}_{2} \mathrm{Cl}_{2}$. Nature Phys. 3, 865-871 (2007).

11. Allan, M. P. et al. Anisotropic energy-gaps of iron-based superconductivity from intra-band quasiparticle interference in LiFeAs. Science 336, 563-567 (2012).

12. Akbari, A., Thalmeier, P. \& Eremin, I. Quasiparticle interference in the

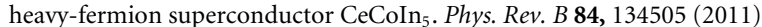

13. Petrovic, C. et al. Heavy-fermion superconductivity in $\mathrm{CeCoIn}_{5}$ at $2.3 \mathrm{~K}$. J. Phys. Condens. Matter 13, L337-L342 (2001).
14. Movshovich, R. et al. Unconventional superconductivity in $\operatorname{CeIrIn}_{5}$ and $\mathrm{CeCoIn}_{5}$ : Specific heat and thermal conductivity studies. Phys. Rev. Lett. 86, 5152-5155 (2001).

15. Izawa, K. et al. Angular position of nodes in the superconducting gap of

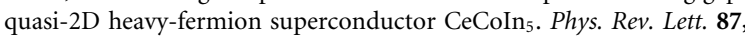
057022 (2001).

16. Stock, C. et al. Spin resonance in the $d$-wave superconductor $\mathrm{CeCoIn}$. Phys. Rev. Lett. 100, 087001 (2008).

17. An, K. et al. Sign reversal of field-angle resolved heat capacity oscillations in a heavy-fermion superconductor $\mathrm{CeCoIn}_{5}$ and $d_{x^{2}-y^{2}}$ pairing symmetry. Phys. Rev. Lett. 104, 037002 (2010).

18. Kenzelmann, M. et al. Coupled superconducting and magnetic order in

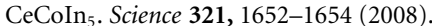

19. $\mathrm{Hu}, \mathrm{T}$. et al. Strong magnetic fluctuations in a superconducting state of

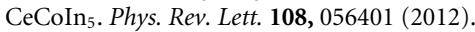

20. Schmidt, A. et al. Imaging the Fano lattice to hidden order transition in $\mathrm{URu}_{2} \mathrm{Si}_{2}$. Nature 465, 570-576 (2010).

21. Yuan, T., Figgins, J. \& Morr, D. K. Hidden order transition in $\mathrm{URu}_{2} \mathrm{Si}_{2}$ Evidence for the emergence of a coherent Anderson lattice from scanning tunneling spectroscopy. Phys. Rev. B 86, 035129 (2012).

22. Aynajian, P. et al. Visualizing heavy fermions emerging in a quantum critical Kondo lattice. Nature 486, 201-206 (2012).

23. Koitzsch, A. et al. Electronic structure of $\mathrm{CeCoIn}_{5}$ from angle-resolved photoemission spectroscopy. Phys. Rev. B 79, 075104 (2009).

24. Jia, X-W. et al. Growth characterization and Fermi surface of heavy-fermion

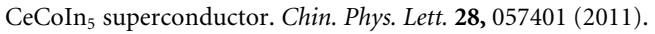

25. Kohori, Y. et al. NMR and NQR studies of the heavy fermion superconductors $\mathrm{CeTIn}_{5}(\mathrm{~T}=\mathrm{Co}$ and Ir). Phys. Rev. B 64, 134526 (2001).

26. Curro, N. J. et al. Anomalous NMR magnetic shifts in CeCoIn ${ }_{5}$. Phys. Rev. B 64, 180514 (2001).

27. Pan, S. H. et al. He-3 refrigerator based very low temperature scanning tunneling microscope. Rev. Sci. Instrum. 70, 1459-1463 (1999).

28. Park, W. K. et al. Andreev reflection in heavy-fermion superconductors and order parameter symmetry in CeCoIn ${ }_{5}$. Phys. Rev. Lett. 100, 177001 (2008).

29. Ernst, S. et al. Scanning tunneling microscopy studies on CeCoIn ${ }_{5}$ and $\mathrm{CeIrIn}_{5}$. Phys. Status Solidi B 247, 624-627 (2010).

30. Tanatar, M. A. et al. Unpaired electrons in the heavy-fermion superconductor $\mathrm{CeCoIn}_{5}$. Phys. Rev. Lett. 95, 067002 (2005).

31. Bianchi, A. D. et al. Superconducting vortices in $\mathrm{CeCoIn}_{5}$ : Toward the Pauli-limiting field. Science 319, 177-180 (2008).

32. Settai, R. et al. Quasi-two-dimensional Fermi surfaces and the de Haas-van Alphen oscillation in both the normal and superconducting mixed states of $\mathrm{CeCoIn}_{5}$. J. Phys. Condens. Matter 13, L627-L634 (2001).

33. McCollam, A. et al. Anomalous de Haas-van Alphen Oscillations in CeCoIn . Phys. Rev. Lett. 94, 186401 (2005).

34. Seyfarth, G. et al. Multigap superconductivity in the heavy-fermion system CeCoIn ${ }_{5}$. Phys. Rev. Lett. 101, 046401 (2008).

\section{Acknowledgements}

We are particularly grateful to I. Eremin, J. E. Hoffman, D-H. Lee and A. R. Schmidt for advice and discussions. We acknowledge and thank A. Akbari, M. Aprili, M. H. Fischer, M. Hamidian, E-A. Kim, S. A. Kivelson, M. Norman, J. P. Reid, D-H. Lee, D. J. Scalapino and K. Shen for helpful discussions, advice and communications. Supported by US DOE under contract number DEAC02-98CH10886 (J.C.D. and C.P.) and under Award No. DE-FG02-05ER46225 (D.K.M., J.v.D.); by the UK EPSRC under programme grant 'Topological Protection and Non-equilibrium States in Correlated Electron Systems' (A.R., A.P.M.); M.P.A. acknowledges support through the ETH Fellowship program; A.P.M. acknowledges support of a Royal Society-Wolfson Award.

\section{Author contributions}

F.M. and M.P.A. carried out SI-STM experiments plus the data preparation; C.P. synthesized and characterized the samples; D.K.M. and J.v.D. developed the band and gap structure models; J.C.D. supervised the project and wrote the paper with key contributions from F.M., M.P.A., D.K.M. and A.P.M. The manuscript reflects the contributions and ideas of all authors.

\section{Additional information}

Supplementary information is available in the online version of the paper. Reprints and permissions information is available online at www.nature.com/reprints. Correspondence and requests for materials should be addressed to D.K.M. or J.C.D.

\section{Competing financial interests}

The authors declare no competing financial interests. 